\title{
PERCUTANEOUS NEPHROLITHOTOMY AND RETRORENAL COLON: ITS INCIDENCE AND COMPLICATIONS
}

S. V. Krishna Reddy ${ }^{1}$, Ahammad Basha Shaik ${ }^{2}$

\section{HOW TO CITE THIS ARTICLE:}

S. V. Krishna Reddy, Ahammad Basha Shaik. "Percutaneous Nephrolithotomy and Retrorenal Colon: Its Incidence and Complications". Journal of Evolution of Medical and Dental Sciences 2015; Vol. 4, Issue 82, October 12; Page: 14329-14334, DOI: 10.14260/jemds/2015/2038

ABSTRACT: OBJECTIVE: The aim of this study was to investigate retrorenal colon incidence in percutaneous nephrolithotomy (PCNL) and to discuss the necessity of routine preoperative CT and perform a cost analysis of this procedure. MATERIALS AND METHODS: Clinical data of 608 PCNL patients over a 5 year period (2008-2013), was surveyed. The patient files were reviewed retrospectively, and only those who had abdominal computed tomography (CT) images before PCNL intervention were included in the study. In the CT images, the position of both the ascending and descending colon in relation to the right and left kidneys were evaluated. We compared the total cost of CTs per incidence of retro colon versus the cost of restoring complications. RESULTS: According to our hospital reports, 262 patients with CT images were included in the present study. 4 patients $(1.14 \%)$ had retrorenal colon, of which $2(0.76 \%)$ were on the left side, $1(0.38 \%)$ on the right side and $1(0.38 \%)$ had bilateral retrorenal colons. Colonic perforation complication was seen only in two patients who had no CT images and the colonic perforation rate was $0.57 \%$. The cost of recognizing one retrorenal colon case using CT was cost-effective to avoid a serious complication. CONCLUSIONS: PCNL, in the process of becoming the standard treatment modality, is a safe and reliable technique for renal stone treatment. Colonic injury should be taken into consideration during PCNL interventions of the lower pole of the kidney. CT, which should be low-dose, if possible, should be performed routinely in all patients to prevent colon injuries. This technique appears to be cost-effective.

KEYWORDS: Percutaneous nephrolithotomy, Retrorenal colon, Complications.

INTRODUCTION: Minimally invasive treatments, such as percutaneous nephrolithotomy (PCNL), retrograde intrarenal surgery (RIRS), non-invasive extracorporeal shock wave lithotripsy (ESWL), and laparoscopy procedures have almost completely replaced open surgery in the management of the urinary stone disease. PCNL which is based on the creation of a suitable percutaneous renal access, dilation of this tract, and fragmentation and elimination of the stone fragments using the nephroscope through an access sheath, is a well-established technique for the treatment of urinary stone in all age groups. Firstly, Goodwin et al.,(1) described the use of a needle to decompress a hydronephrotic kidney. PCNL has become the preferred treatment, especially in cases of large, complex, staghorn calculi, since the first successful stone extraction was performed by a nephrostomy in 1976.(2)

Nowadays, except for the situations including contraindications for general anesthesia, anticoagulant therapy, untreated urinary tract infection, atypical bowel interposition, potential malignant kidney tumour, and pregnancy, PCNL has become a standard modality in the treatment of kidney stones that are larger than $2 \mathrm{~cm}$ in diameter and that do not respond to ESWL.(3) An abdominopelvic ultrasound (USG), plain abdominal films, and intravenous urography are the diagnostic imaging tools to determine stone size, location, and anatomical clues, as well as for 
planning treatment. Moreover, computerised tomography (CT) can be used when there is suspicion of hepatomegaly, splenomegaly, aortic aneurysm and retrorenal colon, allergies of the contrast medium, and in patients with non-opaque stone.(4)

PCNL applications are mostly safe and associated with a low but specific complication rate. These emerge during initial puncture and lead to injuries in the surrounding organs (e.g., colon, pleural cavity, lung, spleen, liver, and duodenum).(5) The most distressing one among these complications are colonic perforations. Though very rare, they may lead to morbidity in the form of septicemia, peritonitis, abscess formation, and nephrocolic or colocutaneous fistula. Hence, knowing the position of the retrorenal colon to prevent surgical complications during PCNL and other surgical modalities is a priority. In the present manuscript, we studied the number of retrorenal colon presence in the CT images taken before PCNL applications.

MATERIALS AND METHODS: The medical records of 608 patients who underwent PCNL in our hospital from March 2008 to November 2013 were reviewed retrospectively. PCNL was conducted in the prone position and under fluoroscopic guidance. Patients' CT images taken before PCNL were studied for the position of both the ascending and descending colon in relation to the right and left kidneys respectively. Patients without CT images and patients with severe musculoskeletal defects, abdominal masses, previous surgery, renal abnormalities (Large renal cysts, ectopic kidney, and horseshoe kidney) and ascites were excluded from the study. Hence, CT images of only 262 cases were included in our study. All CT images taken in supine position were evaluated by the same radiologist for retrorenal colon presence. CT images were as previously identified.(6) to the horizontal plane a parallel line was drawn through the posterior edge of each kidney (posterior renal line) at three renal levels: upper pole, middle pole and lower pole. The number of cases with partial colon extension posterior to the posterior renal line was determined. These were further separated to see if any part of the colon extended directly behind the lower pole [Figures 1], middle pole and upper pole of the kidney.

RESULTS: Of the 262 patients included in the present study, 101 were female and 161 were male with a mean age of 38.2 years. 4 patients $(1.14 \%)$ had retrorenal colon, of which $2(0.76 \%)$ were on the left side, $1(0.38 \%)$ on the right side and $1(0.38 \%)$ had bilateral retrorenal colons. (Table 1$)$. In comparison to kidney location, the retrorenal colons of all patients were at the lower poles. During the studied period, colonic perforation was observed in only 2 cases $(0.3 \%)$ that did not had CT scans taken before PCNL intervention. In our series (262 patients), the cost of recognizing one retrorenal colon case using CT was high for our institution, but because colon injury is a serious complication with a high risk of morbidity and a risk of mortality, an optimal diagnosis should be achieved to avoid this complication.

DISCUSSION: PCNL is a safe and reliable procedure for the treatment of renal stones. However, Michel et al., and de la Rosette et al., reported complication rates of 3\% to 4\% with PCNL as it is an invasive procedure. $(7,8)$ One of the most distressing complications is colonic injury. Although the incidence of this complication is very low (i.e., 0.3 to $1 \%$ of cases) as reported by Skolarikos and de la Rosette, ${ }^{(9)}$ colonic injury may produce dangerous morbidity in the form of septicemia, peritonitis, abscess formation, and nephrocolic or colocutaneous fistula. The risk factors for colon injury include renal anomalies, such as horseshoe kidney, retrorenal colon, previous intestinal bypass surgery, female sex, elderly, thin patients and colonic distension. 
The incidence of colonic injury was also greater on the left side, and a more lateral puncture increases the risk as reported by El-Nahas et al.(10)

Nevertheless, no statistically significant evidence has implicated these factors in the development of colonic injury because these reports contained only small numbers of patients or were single case reports. An essential part of PCNL tract planning is radiological imaging. Intravenous urography (IVU) has historically been the main preoperative imaging technique for PCNL. The spread and increasing presence of multiphase CT scanners has made it the main imaging preference in many centers. IVU however still remains valuable for the preoperative planning and evaluation of kidney stones due to its ability to demonstrate fine details in the collecting system anatomy of the kidney. CT used for the diagnosis of renal stones, also delineates the extent, orientation, and location of the stones within the kidney and thus enable the most suitable PCNL tract selection.

Moreover, by showing the relationship of the kidney and stone to the surrounding structures enables to minimize the risks of injury to the spleen, liver, or to an unsuspected retrorenal colon. A retrorenal colon is more frequently found on the left side and is most likely to be situated near the inferior kidney pole.(11) Retrorenal colon is found in approximatively $0.6 \%$ of the general population according to the Hadar-Gadot and Sherman et al. study.(12,13) In another study with 333 participants, CT images showed that the left colon was posterior in $16.1 \%$ of cases, and the right colon was posterior in $9 \%$ of cases at the level of the lower pole.(14) Total ratio of retrorenal colon was $1.7 \%$, with $1.2 \%$ on the left side, $0.3 \%$ on the right side and $0.2 \%$ bilateral in the Atar et al. study.(15) Hopper et al. reported in their study based on 500 abdomen CT scans that the overall frequency of retrorenal colon was $1.9 \%$ if the images were taken in supine position. When 90 patients were studied in the prone position, retrorenal colon was found in 10\%.(16) In the present study, retrorenal colon in PCNL patients was found in 4 patients $(1.14 \%)$ had retrorenal colon, of which $2(0.76 \%)$ were on the left side, $1(0.38 \%)$ on the right side and $1(0.38 \%)$ had bilateral retrorenal colons.

All CT scans in the present study were performed in supine position. However, in the literature the prevalence of retrorenal colon varies according to the patients' position. Hopper et. al. study, analyzing a series of 500 supine and 90 prone abdominal CT scans, determined the colon positioned posterior to the kidney in $1.9 \%$ and $10 \%$ of cases, respectively. They found that there is a high risk of colon perforation during PCNL in the prone position.(17) If supine and prone positioned patient images are to be compared, the prone position seems to be associated with a significantly shorter nephrostomy tract length and a greater number of potential puncture sites. A shorter tract length may ease percutaneous access and nephroscope mobility within the collecting system and thus improve stone-free rate and decrease hematuria risk.

A greater range of potential access angles may result in decreased risk of visceral injury.(18) In the CROES PCNL study group 1079 PCNL applications were conducted in prone and 232 in supine positions without revealing a difference in complication rates.(19) PCNL was applied a short time ago mainly in endourology clinics; yet, PCNL related complications were generally documented by authors and departments whose main interest is PCNL. However, nowadays PCNL has become a routine practice in all urology clinics and the reported complications may differ from the actual ones.

In the present study, the retrorenal colon rate of $6.9 \%$ of patients who underwent PCNL shows actually how close the urologists are to intervene to a retrorenal colon perforation. Unfortunately, in the two retrorenal colon perforation cases, no CT images were taken before PCNL intervention and thus our ability to evaluate colon perforation risk proactively was nullified On 


\section{ORIGINAL ARTICLE}

the other hand, no colon perforations occurred in any of the cases who had CT images obtained before PCNL intervention.

In order to prevent colon perforation during PCNL, alternative imaging techniques are available. However, a precise and reliable standardization of imaging techniques seems currently not possible. Thus, ultrasound or CT guided puncture to the kidney calyx, in cases with previously reported anatomic variations without inflicting harm to other organs, might be used. Moreover, 3D CT can provide excellent representation of kidney anatomy and be used while planning a PCNL intervention; however, it does not show the relationship between the calices and different organs, such as the colon in particular and kidney motion.(20) Intraoperatively, the visualization of fecal material during nephroscopy or a bowel opacification with nephrostogram is a sign of colon injury.

If this condition is identified intraoperatively, the nephrostomy tube should be replaced into the colon and left as a colonic tube to create a controlled fistula. The urine must be drained with a nephrostomy tube or with a double-J ureteral stent. Broad-spectrum antibiotics must be used. Korkes et al., advocated the colonic tube to be removed when intestinal and urinary tract diversion have been proven.(21) Zuckerman and Desai reported that if in the postoperative period, fecal material in the nephrostomy tube, blood in the feces, pneumaturia, nausea, ileus, vomiting, leukocytosis, fever, sepsis or peritonitis is noted then it should be associated with bowel injury.(22) In the case of extraperitoneal injuries, conservative management may be preferred. The urinary and intestinal tracts must be drained separately via a collecting system with a double-J ureteral stent or a nephrostomy tube. If sepsis or peritonitis is suspected, open surgical repair must be performed.

However, some factors that constrain the implications of our findings should be emphasized. The retrospective nature of the study is a degree of limitation. In addition, the extended study period causes a time effect, with differences and heterogeneity in the study material caused by constant developments in instruments and stone disintegration methods.

CONCLUSIONS: CT is the most commonly used diagnostic method for identifying retrorenal colon location variation. Retrorenal colon is more frequently found on the left side and on the lower pole of the kidney. Therefore, when accessing the lower pole of the kidney, especially on the left side, the risk of colonic injuries should be considered during PCNL. We recommend that despite the low cost of surgery, routine CT should be evaluated in terms of cost and performed to prevent complications that could result in a colostomy and require a series of operations.

\section{REFERENCES:}

1. Goodwin WE et al. Percutaneous trocar (needle) nephrostomy in hydronephrosis. J Am Med Assoc. 1955; 157: 891-4.

2. Fernström I, Johansson B. Percutaneous pyelolithotomy. A new extraction technique. Scand J Urol Nephrol. 1976; 10(3): 257-9.

3. Türk C et al. Guidelines on urolithiasis. European Urological Association. 2014.

4. Altintas $\mathrm{R}$ et al. The importance of instrument type in paediatric percutaneous nephrolithotomy. Urolithiasis. 2014; 42(2): 149-53.

5. Rudnick DM, Stoller ML. Complications of percutaneous nephrostolithotomy. Can J Urol. 1999; 6: 872-5. 
6. Atar M, Hatipoglu NK, Soylemez H, Penbegul N, Bozkurt Y, Gumus H, et al. Relationship between colon and kidney: a critical point for percutaneous procedures. Scand J Urol. 2013; 47: 122-5.

7. Michel, M.S., Trojan, L., Rassweiler, J.J., 2007. Eur. Urol. 51, 899-906; discussion 906.

8. De la Rosette, J.J.M.C.H., Zuazu, J.R., Tsakiris, P., Elsakka, A.M., Zudaire, J.J., Laguna, M.P., de Reijke, T.M., 2008. J. Urol. 180, 2489-2493.

9. Skolarikos, A., de la Rosette, J., 2008. Curr. Opin. Urol. 18, 229-234.

10. El-Nahas, A.R., Shokeir, A.A., El-Assmy, A.M., Shoma, A.M., Eraky, I., El-Kenawy, M.R., ElKappany, H.A., 2006. Urology 67, 937-941.

11. LeRoy AJ, Williams HJ Jr, Bender CE, Segura JW, Patterson DE, Benson RC. Colon perforation following percutaneous nephrostomy and renal calculus removal. Radiology. 1985; 155: 83-5.

12. Hadar H, Gadoth N. Positional relations of colon and kidney determined by perirenal fat. AJR Am J Roentgenol. 1984; 143: 773-6.

13. Sherman JL, Hopper KD, Greene AJ, Johns TT. The retrorenal colon on computed tomography: a normal variant. J Comput Assist Tomogr. 1985; 9: 339-41.

14. Boon JM, Shinners B, Meiring JH. Variations of the position of the colon as applied to percutaneous nephrostomy. Surg Radiol Anat. 2001; 23: 421-5.

15. Atar M, Hatipoglu NK, Soylemez H, Penbegul N, Bozkurt Y, Gumus H, et al. Relationship between colon and kidney: a critical point for percutaneous procedures. Scand J Urol. 2013; 47: $122-5$.

16. Hopper KD, Sherman JL, Luethke JM, Ghaed N. The retrorenal colon in the supine and prone patient. Radiology. 1987; 162: 443-6.

17. Hopper KD, Sherman JL, Williams MD, Ghaed N. The variable anteroposterior position of the retroperitoneal colon to the kidneys. Invest Radiol. 1987; 22: 298-302.

18. Duty B, Waingankar N, Okhunov Z, Ben Levi E, Smith A, Okeke Z. Anatomical variation between the prone, supine, and supine oblique positions on computed tomography: implications for percutaneous nephrolithotomy access. Urology. 2012; 79: 67-71.

19. Astroza G, Lipkin M, Neisius A, Preminger G, De Sio M, Sodha H, et al. CROES PNL Study Group. Effect of supine vs prone position on outcomes of percutaneous nephrolithotomy in staghorn calculi: results from the Clinical Research Office of the Endourology Society Study. Urology. 2013; 82: 1240-4.

20. Goger E, Guven S, Gurbuz R, Yilmaz K, Kilinc M, Ozturk A. Management of a colon perforation during pediatric percutaneous nephrolithotomy. J Endourol. 2012; 26: 1118-20.

21. Korkes, F., Lopes Neto, A.C., Lucio, J., 2nd, Bezerra, C.A., Wroklawski, E.R., 2009. J. Endourol. Endourol. Soc. 23, 569-573.

22. Zuckerman, D.A., Desai, A., 2011. Semin. Interv. Radiol. 28, 438-443.

\begin{tabular}{|c|c|c|c|c|}
\hline Level & Left & Right & Bilateral & Total \\
\hline Upper & 0 & 0 & 0 & 0 \\
\hline Middle & 0 & 0 & 0 & 0 \\
\hline Lower & 2 & 1 & 1 & 4 \\
\hline Total & $\mathbf{2}$ & $\mathbf{1}$ & $\mathbf{1}$ & $\mathbf{4}$ \\
\hline
\end{tabular}

Table 1: Distribution of retrorenal colon according to kidney level 


\section{ORIGINAL ARTICLE}

Figure 1: 64-year-old male with retrorenal colon. Axial enhanced CT images show colon wrapping around lower pole of kidneys and extending posteromedially to psoas muscles.

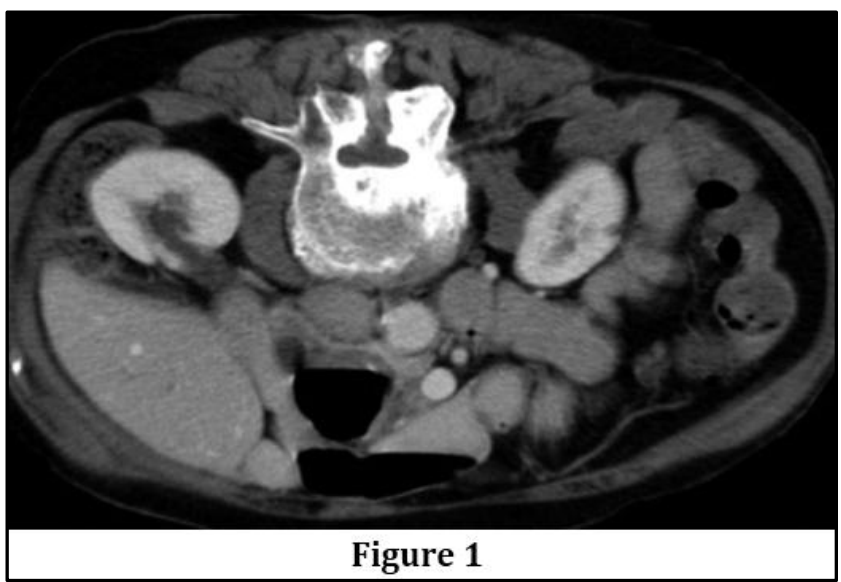

\section{AUTHORS:}

1. S. V. Krishna Reddy

2. Ahammad Basha Shaik

\section{PARTICULARS OF CONTRIBUTORS:}

1. Professor, Department of Urology, Narayana Medical College, Nellore.

2. Assistant Professor, Department of Community Medicine \& Biostatistics Narayana Medical College, Nellore.

\section{FINANCIAL OR OTHER} COMPETING INTERESTS: None
NAME ADDRESS EMAIL ID OF THE CORRESPONDING AUTHOR:

Dr. S. V. Krishna Reddy, Professor, Department of Urology, Narayana Medical College \& Hospital, Nellore-524001, Andhra Pradesh, India.

E-mail: krishnareddysv@narayanagroup.com

Date of Submission: 21/09/2015. Date of Peer Review: 22/09/2015. Date of Acceptance: 03/10/2015. Date of Publishing: 10/10/2015. 